\title{
New Developments in Virtual Reality-Assisted Treatment of Aggression in Forensic Settings: The Case of VRAPT
}

\author{
Fernando Renee González Moraga ${ }^{1,2,3 *}$, Stéphanie Klein Tuente ${ }^{1,2,3}$, Sean Perrin ${ }^{4}$, \\ Pia Enebrink ${ }^{5}$, Kristina Sygel ${ }^{1,3,6}$, Wim Veling ${ }^{7}$ and Märta Wallinius ${ }^{1,2,3}$
}

${ }^{1}$ Research Department, Regional Forensic Psychiatric Clinic, Växjö, Sweden, ${ }^{2}$ Lund Clinical Research on Externalizing and Developmental Psychopathology, Child and Adolescent Psychiatry, Department of Clinical Sciences Lund, Lund University, Lund, Sweden, ${ }^{3}$ Centre for Ethics, Law and Mental Health, Institute of Neuroscience and Physiology, The Sahlgrenska Academy at the University of Gothenburg, Gothenburg, Sweden, ${ }^{4}$ Department of Psychology, Lund University, Lund, Sweden, ${ }^{5}$ Division of Psychology, Department of Clinical Neuroscience, Karolinska Institutet, Stockholm, Sweden, ${ }^{6}$ Department of Forensic Psychiatry, National Board of Forensic Medicine, Stockholm, Sweden, ${ }^{7}$ Department of Psychiatry, University Medical Center Groningen, University of Groningen, Groningen, Netherlands

Aggression is a known problem in individuals being cared for in forensic settings, yet the evidence base for its treatment is scarce. Virtual Reality (VR) has been proposed as a promising addition to interventions in forensic settings, as it may increase the motivation among participants, bridge the gap between real life, therapeutic and laboratory experiences, and increase the ecological validity of psychological research. Recently, a new treatment for aggression using VR as the treatment environment, Virtual Reality Aggression Prevention Training (VRAPT), was developed to provide realistic and safe environments for participants to practice aggression management. In its current revised version, VRAPT is conceptualized as a form of cognitive behavioral therapy with its theoretical background in the General Aggression Model. Its purpose is to increase awareness of, and improve control over, one's own aggression and that of others through social interactions in individually tailored virtual environments. This manuscript describes how the lessons learned from the first randomized controlled trial of VRAPT have been applied to further develop the method and discusses challenges and future directions for VR-assisted treatment of aggression in forensic settings. VRAPT is a new psychological treatment for aggression and the coming years will provide expanded scientific evidence for further developments and adaptations.

Keywords: aggression, virtual reality, forensic settings, VRAPT, treatment of aggression, forensic psychiatry

\section{INTRODUCTION}

Human aggression has been defined as: “...deliberate and directed behavior with an immediate intention to harm another individual and the other individual is motivated to avoid this behavior" (Anderson and Bushman, 2002, p. 28). Physical violence is defined as a (more extreme) form of aggression. In short, violent behavior is always aggression, while most forms of aggression (e.g., verbal threats) are not physical violence (Warburton and Anderson, 2015). Aggression in the form of interpersonal violence is a major challenge to society, causing suffering to victims and their families 
as well as significant direct and indirect costs to society (Mikton et al., 2016; Hoeffler, 2017). In general, a small number of offenders account for the majority of violent crimes (Falk et al., 2014) and are subjected to incarceration in forensic settings (e.g., prisons, forensic psychiatric care, young offender institutions) with varying availability of evidence-based interventions. ${ }^{1}$ Violent offenders constitute a heterogeneous group, with some demonstrating very complex psychiatric comorbidities (e.g., forensic psychiatric patients) and others predominantly criminogenic needs. However, common denominators for violent offenders housed in forensic settings are difficulties with emotion regulation, impulsivity, and empathy in combination with different mental disorders (e.g., antisocial and other personality syndromes) (Bogaerts et al., 2012; Lobbestael et al., 2015; Filov, 2019; Mundt and Baranyi, 2020). Given this, maintaining an environment that reduces the risk of aggression represents a distinct challenge. This is true both for the safety of the residents and the staff and engaging in and being exposed to aggression in forensic settings is associated with an increased risk of reoffending after discharge (Needham et al., 2004; Dack et al., 2013; Mooney and Daffern, 2015). Thus, it is of utmost importance to identify methods to effectively treat individuals with aggression problems in forensic settings (González Moraga et al., 2019).

Regarding aggression treatment, a recent meta-analytic review identified 27 controlled studies carried out in forensic settings and found that multimodal, psychological treatments were effective in reducing both violent $(0.27 \geq \mathrm{OR} \leq 0.69, p<0.05)$ and general/ nonviolent $(0.19 \geq \mathrm{OR} \leq 0.75, p<0.05)$ recidivism in adult violent offenders (Papalia et al., 2019). In sum, treatments significantly reduced violent recidivism by $10.2 \%$ points and nonviolent/ general recidivism by $11.2 \%$ points for violent offenders receiving treatment, relative to violent offenders not receiving treatment. However, firm conclusions about the efficacy of these approaches are hampered by high levels of heterogeneity (e.g., in treatment components, outcome measures, study populations) in the identified studies and more well-designed trials of theory- and data-driven treatments for aggression are needed (Lee and DiGiuseppe, 2018). In addition, further efforts are needed to identify therapeutically active components in multi-component treatments, as well as mechanisms that underpin change.

There are several aspects in need of consideration to increase the efficacy of aggression treatments. Standard evidence-based treatment programs for aggression involve a variety of cognitive and behavioral interventions that target threat biases through cognitive restructuring, training in emotion (particularly anger) and arousal regulation skills, and role-plays in which the individual practices skills that have the overall aim of reducing the likelihood of aggressive behaviors (Lee and DiGiuseppe, 2018). As the expression of aggressive behaviors is influenced by individual characteristics and the context in which they are

\footnotetext{
${ }^{1} \mathrm{~A}$ recent systematic review (Howner et al., 2018) demonstrated profound knowledge gaps in all areas identified as important for forensic psychiatric care, including diagnostic and risk assessments, psychological interventions and rehabilitation.
}

triggered (Griskevicius et al., 2009), the treatment must involve identification of, and exposure to (during role-plays), the kinds of triggers/situations that are most relevant to that individual. Personalizing treatment in this way brings with it certain difficulties, particularly in forensic settings, where strict designs to prevent violence are often in place, recreating contexts that may trigger aggression in a specific individual as part of a therapeutic role-play can be extremely challenging and may have an increased risk of physical harm to the therapist, the individual or observers (e.g., in group-based treatments). In forensic settings, Virtual Reality (VR) holds the promise of providing a safer and more personalized method for identifying idiosyncratic cues for anger and aggression and modifying the individual's responses to these cues, including through role-plays (Bouchard and Rizzo, 2019). Thus, there is a need for further developments and research incorporating VR technology into the treatment of aggression.

This manuscript will provide a brief overview of the treatment of human aggression and discuss how VR can be implemented to support such treatment. Recent progress and developments with a newly established treatment for aggression, Virtual Reality Aggression Prevention Training VRAPT (Tuente et al., 2018; Klein Tuente et al., 2020), will be described along with challenges in, and future directions for, VR-assisted treatment of aggression in forensic settings.

\section{TREATING DYSFUNCTIONAL AGGRESSION IN FORENSIC SETTINGS}

To develop an effective psychological intervention for aggression, it is important to identify the triggers, motives and functions of the aggressive behaviors (Tapscott et al., 2012; Lobbestael et al., 2015), along with incentives to change (e.g., improvements in emotion and behavior regulation, interpersonal functioning, and goal attainment). Lewis and Ireland (2019) described four areas crucial to the understanding of motives for aggression in forensic psychiatric patients; patients' (perceived need for) protection, social recognition (e.g., aggression to establish/confirm a role in social hierarchies), emotion regulation (e.g., emotional release through aggressive outbursts), and communication (e.g., expressing frustration or dissatisfaction), all of which can be targeted in cognitive behavioral programs aimed at anger and aggression reduction. A recent meta-analysis by Papalia and others (2019) found that intensive multimodal cognitive behavioral therapy (CBT) programs that incorporate relapse prevention, role-plays, home assignments, interpersonal skills training, and anger control provided larger reductions in violent recidivism than treatments that do not include these components. Examples of such CBT programs include Aggression Replacement Training ART (Goldstein et al., 1998) and Responsive Aggression Regulation Therapy Re-ART (Hoogsteder et al., 2014). ART, originally developed for aggressive youth but later used also in adult groups (Brännström et al., 2016), is based on three components: Skillstreaming with role-plays on social interactions over different skill areas, Anger Control Training including 
modeling, role-playing, and performance feedback to increase awareness and management of aggression triggers, and Moral Reasoning Training comprising structured discussions on different moral dilemmas. All ART sessions are given over in total 10 weeks (one weekly session for each component) to peer groups led by ART trainers. Re-ART, intended for adolescents and young adults with severe aggression problems and available both for residential and outpatient settings, is an individual treatment provided one to three times a week and can be given as a compact version (4 months) and a complete version (10 months) (Schippers et al., 2020). Re-ART is based on four modules (Intake and Motivation, Controlling Skills, Influence of Thinking, Assertiveness) with optional modules for Stress Reduction, Impulse Control, Observation and Interpretation, Emotion Regulation, Handling Conflicts, and Family. Even though Re-ART is an individual treatment, in contrast to ART's peer group design, the system (e.g., family) is involved in the treatment when possible, to support the individual's learning. However, when considering the evidence-base for aggression treatments, it must be noted that the knowledge on long-term effects of treatment is scarce, with many studies having follow-up periods for a maximum of 12 months (Papalia et al., 2019). Furthermore, no specific aggression treatments for individuals with aggression and severe forms of mental illness involving neurocognitive deficits (e.g., schizophrenia) have been evaluated. In addition, the effect sizes for the existing treatments often fall in the small range with high rates of recidivism being common (Watt and Howells, 1999; Hornsveld et al., 2008; Brännström et al., 2016; Papalia et al., 2019).

In summary, the challenges for interventions in forensic settings are many. Low treatment motivation and treatment dropout is common which may help explain the limited efficacy of psychological interventions for aggression and high rates of recidivism (Dixon et al., 2016; Kip et al., 2018; Brunner et al., 2019). While there is some evidence that low motivation and dropout are associated with higher levels of psychopathic traits, proactive aggression, and more weekly substance use (Smeijers et al., 2018), in general there is a poor understanding of treatment moderators (i.e., factors which help to explain which treatment works best for whom) and mediators (i.e., mechanisms that underpin improvements in treatment). Furthermore, a specific challenge can be seen in interventions being carried out in secluded environments with little or no resemblance to the contexts that are relevant for an individual's aggression management. Thus, a need for increased ecological validity of aggression treatments in forensic settings is evident.

\section{VIRTUAL REALITY IN FORENSIC SETTINGS}

In forensic settings, eHealth applications including VR have been presented as possible methods to increase treatment motivation among participants through using innovative, state-of-the-art technologies that appeal to participants and increase their perceived personal relevance of the intervention (Kip et al., 2018). Interventions involving VR have been particularly emphasized as promising eHealth applications in forensic settings (Fromberger et al., 2014), and have been suggested to bridge the gap between real life and laboratory experiences and thus increase the ecological validity of psychological research (Kisker et al., 2019). A recent systematic review (Sygel and Wallinius, 2021) demonstrated promising developments in adjacent fields (e.g., treatment of psychosis), however only a few methods, focusing on simulations of realistic offenserelated stimuli in the assessment and treatment of deviant sexual interests (Renaud et al., 2005; Benbouriche et al., 2014; Renaud et al., 2014; Fromberger et al., 2018), have been evaluated for forensic settings so far. In relation to aggression, only two methods employing VR have been described: the VR Game for Aggressive Impulse Management VR-GAIME (Smeijers and Koole, 2019), developed as a complement to ART interventions, and the VR Aggression Prevention Training for forensic psychiatric patients (VRAPT; Tuente et al., 2018; described in detail below). So far, the evidence for these approaches remains extremely limited.

VR-GAIME follows the philosophy of a "serious game" (i.e., the primary purpose is training, education, or health improvement) and has been developed as an avoidance motivation intervention for anger management that is fun and challenging to participants (Smeijers and Koole, 2019). During the game, the participant is working as a mail courier with the task of collecting lost packages by walking down a shopping street. In the shopping street, the participant is met by avatars who act in either an agreeable or disagreeable manner. Participants are instructed to lean forward (i.e., make an approach movement) in response to agreeable avatars and to lean backwards (i.e., make an avoidance movement) in response to disagreeable avatars, thus priming participants to respond with avoidance behavior to anger-invoking situations. VR-GAIME has five levels with ascending difficulty levels, and the participant can proceed to the next level once enough packages have been collected. The first randomized controlled trial of VR-GAIME as a complement to ART for forensic psychiatric patients is ongoing.

\section{RECENT DEVELOPMENTS AND CURRENT ADVANCES IN VR-ASSISTED TREATMENT OF AGGRESSION IN FORENSIC SETTINGS: THE CASE OF VRAPT}

VRAPT was developed in the Netherlands as a collaboration between researchers and clinicians to create a structured, psychological VR-assisted treatment for forensic psychiatric patients with aggression problems (Tuente et al., 2018). The first versions of VRAPT were based on the social information processing model SIP (Crick and Dodge, 1994), focusing on disturbances in social information processing (e.g., hostile attribution bias) as an explanation for aggressive behaviors (Tuente et al., 2018; Klein Tuente et al., 2020). In the VRenvironment, each step of a simplified SIP model (1. Encoding: What is going on? 2. Interpretation: What does this mean? 3. Selecting a goal: What is the goal I'm trying to achieve in 
this situation? 4. Generating responses: How can I react to this? 5. Evaluating responses: What am I going to do? 6. Enacting responses: Reaction/Behavior) was practiced through social scenarios with varying degrees of direct interaction between participant and therapist over 16 sessions, $45-60 \mathrm{~min}$ in length, delivered once or twice a week. The first five sessions focused on practicing emotion recognition skills in the VR environment, while sessions 6 to 15 were designed to help the patients practice regulating their own and other's aggression during role-plays in virtual environments. From session 6 onward, physiological measures (i.e., skin conductance and heart-rate variability) were added to provide real-time physiological feedback to the patient and therapist during the interactive VR role-plays. All role-plays were therapist-led, with the aim of facilitating real-time tailoring of the virtual environment and interactions to the patient. Thus, the participant's aggression could be observed and managed through real-time therapeutic intervention and the treatment tailored to the specific needs of the participant, e.g., specific situations and skills.

VRAPT was recently evaluated in a multi-site RCT, which indicated that VRAPT influenced anger control skills, impulsivity, and hostility (secondary outcomes) in forensic psychiatric patients post-treatment but not at follow-up, and with no statistically significant effect of reduction of aggressive behaviors (primary outcome) as reported by the patients themselves or through staff observations (Klein Tuente et al., 2020). In relation to the aims of VRAPT, the RCT demonstrated that treatment of aggression using VR was possible in forensic settings, but that revisions of the method, and possibly also of the tools used in the evaluation of its effectiveness, were needed.

Consequently, VRAPT has been revised and is currently being tested in pilot studies in forensic psychiatric and prison settings. The target group remains the same; predominantly individuals in forensic settings with under- or overregulation problems of reactive aggression. VRAPT in its revised version is conceptualized as a manualized, CBT approach for aggression treatment, with a theoretical underpinning that has been extended from the SIP model to the broader General Aggression Model GAM (Anderson and Bushman, 2002; Anderson and Carnagey, 2004; DeWall et al., 2011) to provide a more comprehensive understanding of the needs of the specific participant considering contextual factors. The GAM is a comprehensive, social-cognitive, psychological theory in which human aggression arises from a combination of innate conditions (e.g., genetic and other biological factors), early life circumstances, development and maintenance of aggression-related beliefs, and exposure to situational factors that trigger these vulnerabilities (e.g., provocation, pain) and increase the likelihood of aggressive behaviors. The GAM also describes how a person, through increased awareness and cognitive control, can better manage their aggressive impulses and engage in more adaptive behaviors in response to provocations (Barlett and Anderson, 2011; Sestir and Bartholow, 2007). In VRAPT, a simplified illustration of the GAM has been adapted for use with patients during sessions (see Figure 1), to guide the patient in the conceptualization process. Introducing the GAM into VRAPT has led to changes in the content of all treatment sessions in comparison to previous versions, with a broader focus on both individual and contextual vulnerabilities that are considered relevant to increase the intervention's efficacy. Furthermore, VRAPT increases its emphasis on the roles played by emotion recognition and regulation in the etiology and treatment of aggression. In summary, the theoretical underpinning of the revised VRAPT differs from the previous versions in an outspoken CBT approach and a broader conceptualization of aggression through the GAM model.

The aim of VRAPT is to increase the participant's understanding and management of his/her dysfunctional aggressive behaviors through identifying triggers and risk scenarios in a conceptualization with assistance of the VRAPT model presented in Figure 1. VRAPT is divided into four treatment modules; A-D (see Table 1). Module A introduces the VRAPT model, focuses on treatment conceptualization, and lets the participant experience the virtual environment for the first time through walking around in one or several virtual environments. Module B entails assignments in the virtual world where the participant is tasked with assessing and discerning emotions in avatars as expressed in facial expressions and practicing recognition and management of own physiological reactions when confronted with an aggression-provoking role-play in the virtual environment. ${ }^{2}$ In Module C (Skills Training), the participant practices social skills and management of own and others' aggression through individually tailored, therapist-led role-plays in the VRenvironment where the therapist plays the avatars in the virtual environment using voice distortion and triggering behavioral responses of the avatar. During parts of Module B and throughout Module C, physiological measures (e.g., heartrate variability and skin conductance) are used to assist therapist and participant in recognizing physiological reactions indicating increased physiological arousal in the participant. Finally, Module D (Evaluation) summarizes the learning outcomes from the VRAPT intervention. Module A-C is to a large extent carried out in the virtual environment provided by the software Social Worlds (CCleVR), with the participant interacting with avatars available in the virtual environment. The virtual environments are set up by the therapist before the session through a simple choice menu, with many different social environments (e.g., shopping street, café, ward, school), avatars (different ethnicities, ages, and the total number of avatars moving in the environment) and environmental sounds (e.g., police car, barking dogs, crying children) to choose from. In comparison to previous versions, the revised VRAPT emphasizes generalization of skills to the participant's current contexts through involving staff.

${ }^{2}$ Please see the link for screenshots of Social Worlds software (https:/clevr.net/ producten.html). 


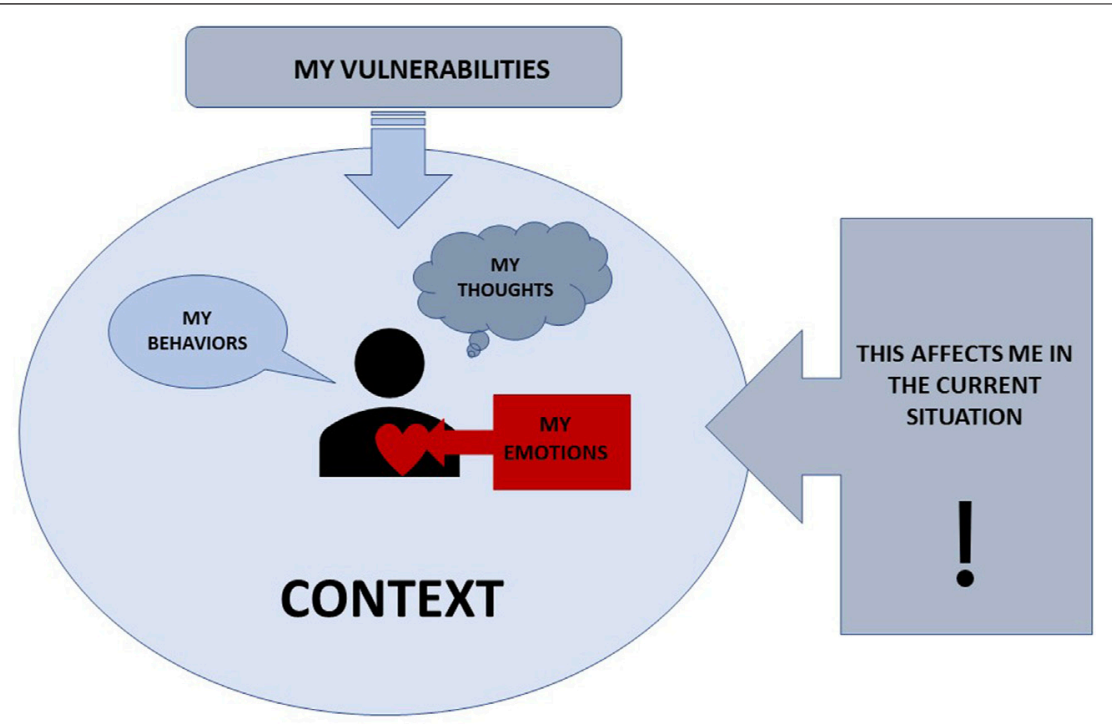

FIGURE 1 | The VRAPT model.

TABLE 1 | Overview of the VRAPT.

VRAPT module Session(s)

A. Introduction

1

Providing information on the
VRAPT

Familiarize with the virtual environment

B. Assessment

2-6

Assessing treatment needs Formulating treatment goals Practicing recognition and discern of emotions

Practicing recognition of physical tension preceding aggression Identifying relaxation techniques

C. Skills Training 6-15

Skills training in de-escalation strategies (VR role-plays) Practicing relaxation techniques to reduce own physical tension
Increase knowledge on the concept and development of aggression

Increase motivation to participate in the VRAPT

Conceptualization of the participant's needs in relation to aggression management

Clear, individually tailored treatment goals

Increased knowledge on the importance of, and skills in, recognizing and discerning emotions and physical tension preceding aggression Increased knowledge on, and skills in, techniques to reduce own physical tension

Increased knowledge on risk scenarios for aggression

Increased knowledge on, and skills in, de-escalation of aggression

Increased knowledge on, and skills in, techniques to reduce own physical tension
Interaction in social world ${ }^{1}$ (VR environment)
The participant can "walk around" in 5 different virtual environments: shopping street, supermarket, restaurant, buss or a park with the guidance of the therapist

Emotion recognition on a virtual shopping street where the participant, with the guidance of the therapist, is tasked with naming emotions of different avatars in the virtual environment as demonstrated by their facial expressions. This task is divided into two parts: 1. Recognizing emotions, 2. Discerning emotions

The participant interacts in VR role-plays led by the therapist. The role-plays are decided beforehand in collaboration between therapist and participant, based on treatment needs and specific risks for the participant. In every single VR environment, the participant has the possibility to interact with one or more avatars, played by the therapist

D. Evaluation $\quad 16 \quad$ Evaluation of the participant's Evaluation of progress in relation to treatment goals N/A experiences of the VRAPT.

Social Worlds ${ }^{\circledR}$ is provided by the software company CleVR and is used for all VR tasks in VRAPT.

\section{CHALLENGES AND ADVANTAGES IN VR-ASSISTED TREATMENT OF AGGRESSION IN FORENSIC SETTINGS}

VRAPT is a newly developed method for treating aggression that has shown some promise in a recent RCT involving inpatients on forensic psychiatric units (Klein Tuente et al., 2020). Importantly, and despite the use of repeated VR-assisted role-plays involving exposure to provocations for aggression, the investigators found no evidence that participation in VRAPT increased the levels, or expressions, of aggression by the treated patients or towards them by other patients on the units. This is important because there is 
some evidence that participation in aggression-focused treatments involving role-plays by inpatients on forensic units can be associated with an increased risk of aggression on the unit and after discharge (Danielsson et al., 2011).

Specific advantages of VR-assisted aggression treatment in comparison to previous methods involving role-plays is that VR allows greater collaboration and feedback (including physiological) between the therapist and participant so that the role-plays can be better tailored to the participant's specific needs, thus allowing them to practice situations which provide a challenge but are still possible to manage without resorting to violence or aggression. In this way, participants should gain increased awareness of their own "triggers" for anger and aggression, and practice different methods for downregulating their emotions, problem-solving, and engaging in pro-social behaviors. Importantly, as in all interactive role-plays, these can be interrupted by either the participant or the therapist at any time. The therapist can then support the participant in the way needed, e.g. shifting their attention to different physiological cues for arousal/anger, prompting relaxation techniques (e.g., breathing), identifying and modifying aggression-related appraisals in relation to interoceptive and situational cues, and problem-solving different approaches to reduce the likelihood of an aggressive or violent response. This can be done both within the virtual environment and in the clinical environment when the participant removes the VR headset.

Challenges in VR-assisted aggression treatment bear similarities to VR methods in general. A known side-effect of using a VR headset is so called "cybersickness," a type of motion sickness that may cause a person can become dizzy and feel unwell while in the virtual environment (Weech et al., 2019). If this happens during VRAPT, the therapist will guide the participant through specific strategies to feel better and, if this does not work, abort the ongoing session and jointly evaluate if continued VRAPT treatment is feasible, given the participant's experiences. Specific challenges in the VRAPT include ethical challenges (World Medical Association, 2013; European Science Foundation and All European Academies, 2011). Individuals in forensic settings are deprived of their liberty, and a vast majority suffer from varying degrees of mental disorders, with forensic psychiatric patients demonstrating the most severe mental illnesses (Munthe et al., 2010; Shapiro, 2016; Völlm et al., 2016). Thus, it is crucial to avoid therapeutic misconception, such that the participant believes that research participation is part of the current care process (Appelbaum et al., 2012; Appelbaum and Lidz, 2012). Due to the nature of VRAPT as an aggression treatment, security considerations are also important to provide a safe environment for patients, clinicians, and researchers. Even though the level of difficulty increases gradually over the sessions, participants who pose a serious, current risk to the safety of themselves or others (e.g., a risk of severe violence that cannot be handled during the

\section{REFERENCES}

Anderson, C. A., and Bushman, B. J. (2002). Human Aggression. Annu. Rev. Psychol. 53 (1), 27-51. doi:10.1146/annurev.psych.53.100901.135231 treatment sessions) must be excluded from participation. Importantly, as most of the current target population for VRAPT is under compulsory forensic psychiatric care or within the prison system, around-the-clock support from staff is available and should be integrated into treatment delivery.

\section{CONCLUSION}

Individuals being cared for in forensic settings constitute a particularly vulnerable group in society. There is a lack of evidence-based treatment methods, particularly for aggression, for use in these settings (Howner et al., 2018; Lee and DiGiuseppe, 2018). Hence, the need for studies in this area is urgent. In this manuscript, we have presented a brief overview of human aggression and its treatment, and using the example of VRAPT, explained how VR-assisted interventions may represent a step forward in the development of safer, ecologically valid, and effective interventions for aggression in forensic settings. Significant challenges still lay ahead, including the feasibility of a treatment that places relatively high demands on the therapist and patient alike to use the wealth of data provided during VR-assisted sessions to tailor the treatment effectively. While the acceptability, feasibility, and efficacy of programs like VRAPT require further investigation, VR, and extended reality (XR) technologies in general, provide completely new possibilities for improving development of more effective approaches for aggression for use in forensic settings.

\section{DATA AVAILABILITY STATEMENT}

The original contributions presented in the study are included in the article/Supplementary Materials, further inquiries can be directed to the corresponding authors.

\section{AUTHOR CONTRIBUTIONS}

FG, MW and SKT contributed to conception of the manuscript. FG wrote the first draft of the manuscript. SKT, SP, PE, KS, and MW wrote sections of the manuscript. All authors contributed to manuscript revisions, read and approved the submitted version.

\section{FUNDING}

This work was supported by the Swedish Research Council for Health, Working Life and Welfare under Grant No. 2018-01409, by the Southern Healthcare Region in Sweden (No. 933868), and by Region Kronoberg (No. 936434).
Anderson, C. A., and Carnagey, N. L. (2004). Violent Evil and the General Aggression Model. The Soc. Psychol. Good Evil, 168-192.

Appelbaum, P. S., Anatchkova, M., Albert, K., Dunn, L. B., and Lidz, C. W. (2012). Therapeutic Misconception in Research Subjects: Development and Validation of a Measure. Clin. Trials 9 (6), 748-761. doi:10.1177/1740774512456455 
Appelbaum, P. S., and Lidz, C. W. (2012). The Mismeasure of Therapeutic Misconception. Clin. Trials 9 (6), 765-766. doi:10.1177/1740774512464312

Barlett, C. P., and Anderson, C. A. (2011). Reappraising the Situation and its Impact on Aggressive Behavior. Pers Soc. Psychol. Bull. 37 (12), 1564-1573. doi:10.1177/0146167211423671

Benbouriche, M., Nolet, K., Trottier, D., and Renaud, P. (2014). "April). Virtual Reality Applications in Forensic Psychiatry," in Proceedings of the 2014 Virtual Reality International Conference forensic psychiatry. Paper Presented at the Proceedings of the 2014 Virtual Reality International Conference, France (Laval), 1-4.

Bogaerts, S., Polak, M., Spreen, M., and Zwets, A. (2012). High and Low Aggressive Narcissism and Anti-social Lifestyle in Relationship to Impulsivity, Hostility, and Empathy in a Group of Forensic Patients in the Netherlands. J. Forensic Psychol. Pract. 12 (2), 147-162. doi:10.1080/15228932.2012.650144

Bouchard, S., and Rizzo, A. A. (2019). Virtual Reality for Psychological and Neurocognitive Interventions. Berlin: Springer.

Brännström, L., Kaunitz, C., Andershed, A. K., South, S., and Smedslund, G. (2016). Aggression Replacement Training (ART) for Reducing Antisocial Behavior in Adolescents and Adults: A Systematic Review. Aggression Violent Behav. 27, $30-41$.

Brunner, F., Neumann, I., Yoon, D., Rettenberger, M., Stück, E., and Briken, P. (2019). Determinants of Dropout from Correctional Offender Treatment. Front. Psychiatry 10, 142. doi:10.3389/fpsyt.2019.00142

Crick, N. R., and Dodge, K. A. (1994). A Review and Reformulation of Social Information-Processing Mechanisms in Children's Social Adjustment. Psychol. Bull. 115 (1), 74-101. doi:10.1037/0033-2909.115.1.74

Dack, C., Ross, J., Papadopoulos, C., Stewart, D., and Bowers, L. (2013). A Review and Meta-Analysis of the Patient Factors Associated with Psychiatric In-Patient Aggression. Acta Psychiatrica Scand. 127 (4), 255-268. doi:10.1111/acps.12053

Danielsson, M., Fors, A., and Freij, I. (2011). Behandlingsprogrammet ART I Kriminalvården-Utvärdering Av Återfall I Brott För Programdeltagare 2003-2006. Norrköping: Kriminalvården.

DeWall, C. N., Anderson, C. A., and Bushman, B. J. (2011). The General Aggression Model: Theoretical Extensions to Violence. Psychol. Violence 1 (3), 245-258. doi:10.1037/a0023842

Dixon, L. B., Holoshitz, Y., and Nossel, I. (2016). Treatment Engagement of Individuals Experiencing Mental Illness: Review and Update. World Psychiatry 15 (1), 13-20. doi:10.1002/wps.20306

European Science Foundation, and All European Academies (2011). The European Code of Conduct for Research Integrity. European Science Foundation.

Falk, O., Wallinius, M., Lundström, S., Frisell, T., Anckarsäter, H., and Kerekes, N. (2014). The $1 \%$ of the Population Accountable for $63 \%$ of All Violent Crime Convictions. Soc. Psychiatry Psychiatr. Epidemiol. 49 (4), 559-571. doi:10.1007/ s00127-013-0783-y

Filov, I. (2019). Antisocial Personality Traits as a Risk Factor of Violence between Individuals with Mental Disorders. Open Access Maced J. Med. Sci. 7 (4), 657-662. doi:10.3889/oamjms.2019.146

Fromberger, P., Jordan, K., and Müller, J. L. (2014). Anwendung virtueller Realitäten in der forensischen Psychiatrie. Nervenarzt 85 (3), 298-303. doi:10.1007/s00115-013-3904-7

Fromberger, P., Jordan, K., and Müller, J. L. (2018). Virtual Reality Applications for Diagnosis, Risk Assessment and Therapy of Child Abusers. Behav. Sci. L. 36 (2), 235-244. doi:10.1002/bsl.2332

Goldstein, A. P., Glick, B., and Gibbs, J. C. (1998). Aggression Replacement Training: A Comprehensive Intervention for Aggressive Youth. Rev. Research Press.

González Moraga, F. R., Garcia, D., Billstedt, E., and Wallinius, M. (2019). Facets of Psychopathy, Intelligence, and Aggressive Antisocial Behaviors in Young Violent Offenders. Front. Psychol. 10, 984. doi:10.3389/fpsyg.2019.00984

Griskevicius, V., Tybur, J. M., Gangestad, S. W., Perea, E. F., Shapiro, J. R., and Kenrick, D. T. (2009). Aggress to Impress: Hostility as an Evolved Contextdependent Strategy. J. Personal. Soc. Psychol. 96 (5), 980-994. doi:10.1037/ a0013907

Hoeffler, A. (2017). What Are the Costs of Violence. Polit. Philos. Econ. 16 (4), 422-445. doi:10.1177/1470594x17714270

Hoogsteder, L. M., Kuijpers, N., Stams, G. J. J. M., E. van Horn, J., Hendriks, J., and Wissink, I. B. (2014). Study on the Effectiveness of Responsive Aggression
Regulation Therapy (Re-ART). Int. J. Forensic Ment. Health 13 (1), 25-35. doi:10.1080/14999013.2014.893711

Hornsveld, R. H. J., Nijman, H. L. I., and Kraaimaat, F. W. (2008). Aggression Control Therapy for Violent Forensic Psychiatric Patients: First Results. Psychol. Crime L. 14 (1), 1-18. doi:10.1080/10683160701340569

Howner, K., Andiné, P., Bertilsson, G., Hultcrantz, M., Lindström, E., Mowafi, F., et al. (2018). Mapping Systematic Reviews on Forensic Psychiatric Care: a Systematic Review Identifying Knowledge Gaps. Front. Psychiatry 9, 452. doi:10.3389/fpsyt.2018.00452

Kip, H., Bouman, Y. H. A., Kelders, S. M., and van Gemert-Pijnen, L. J. E. W. C. (2018). eHealth in Treatment of Offenders in Forensic Mental Health: a Review of the Current State. Front. Psychiatry 9, 42. doi:10.3389/fpsyt.2018.00042

Kisker, J., Gruber, T., and Schöne, B. (2019). Experiences in VR Entail Different Processes of Retrieval as Opposed to Conventional Laboratory Settings: A Study on Human Memory. Curr. Psychol., 1-8.

Klein Tuente, S., Bogaerts, S., Bulten, E., Keulen-de Vos, M., Vos, M., Bokern, H., et al. (2020). Virtual Reality Aggression Prevention Therapy (VRAPT) versus Waiting List Control for Forensic Psychiatric Inpatients: a Multicenter Randomized Controlled Trial. Jcm 9 (7), 2258. doi:10.3390/jcm9072258

Lee, A. H., and DiGiuseppe, R. (2018). Anger and Aggression Treatments: a Review of Meta-Analyses. Curr. Opin. Psychol. 19, 65-74. doi:10.1016/ j.copsyc.2017.04.004

Lewis, M., and Ireland, J. L. (2019). Understanding Motives for Aggression in Forensic Psychiatric Patients: a Preliminary Study. J. Forensic Psychiatry Psychol. 30 (3), 496-509. doi:10.1080/14789949.2019.1570541

Lobbestael, J., Cima, M., and Lemmens, A. (2015). The Relationship between Personality Disorder Traits and Reactive versus Proactive Motivation for Aggression. Psychiatry Res. 229 (1-2), 155-160. doi:10.1016/ j.psychres.2015.07.052

Mikton, C. R., Butchart, A., Dahlberg, L. L., and Krug, E. G. (2016). Global Status Report on Violence Prevention 2014. Am. J. Prev. Med. 50 (5), 652-659. doi:10.1016/j.amepre.2015.10.007

Mooney, J. L., and Daffern, M. (2015). The Relationship between Aggressive Behaviour in Prison and Violent Offending Following Release. Psychol. Crime L. 21 (4), 314-329. doi:10.1080/1068316x.2014.989163

Mundt, A. P., and Baranyi, G. (2020). The Unhappy Mental Health Triad: Comorbid Severe Mental Illnesses, Personality Disorders, and Substance Use Disorders in Prison Populations. Front. Psychiatry 11, 804. doi:10.3389/ fpsyt.2020.00804

Munthe, C., Radovic, S., and Anckarsã "Ter, H. (2010). Ethical Issues in Forensic Psychiatric Research on Mentally Disordered Offenders. Bioethics 24 (1), 35-44. doi:10.1111/j.1467-8519.2009.01773.x

Needham, I., Abderhalden, C., Meer, R., Dassen, T., Haug, H. J., Halfens, R. J. G., et al. (2004). The Effectiveness of Two Interventions in the Management of Patient Violence in Acute Mental Inpatient Settings: Report on a Pilot Study. J. Psychiatr. Ment. Health Nurs. 11 (5), 595-601. doi:10.1111/j.13652850.2004.00767.x

Papalia, N., Spivak, B., Daffern, M., and Ogloff, J. R. (2019). A Meta-analytic Review of the Efficacy of Psychological Treatments for Violent Offenders in Correctional and Forensic Mental Health Settings. Clin. Psychol. Sci. Pract. 26 (2), e12282. doi:10.1111/cpsp.12282

Renaud, P., Proulx, J., Rouleau, J. L., Bouchard, S., Madrigrano, G., Bradford, J., et al. (2005). The Recording of Observational Behaviors in Virtual Immersion: A New Clinical Tool to Address the Problem of Sexual Preferences with Paraphiliacs. Annu. Rev. Cybertherapy Telemedecine 3, 85-92.

Renaud, P., Trottier, D., Rouleau, J.-L., Goyette, M., Saumur, C., Boukhalfi, T., et al. (2014). Using Immersive Virtual Reality and Anatomically Correct ComputerGenerated Characters in the Forensic Assessment of Deviant Sexual Preferences. Virtual Reality 18 (1), 37-47. doi:10.1007/s10055-013-0235-8

Schippers, E. E., Hoogsteder, L. M., and Stams, G. J. J. M. (2020). Responsive Aggression Regulation Therapy (Re-ART) Improves Executive Functioning in Adolescents and Young Adults with Severe Aggression Problems: A Pilot Study. J. Forensic Sci. 65 (6), 2058-2064. doi:10.1111/1556-4029.14539

Sestir, M. A., and Bartholow, B. D. (2007). Theoretical Explanations of Aggression and Violence. Aggressive Offenders Cognition. Theor. Res. Pract., 157-178.

Shapiro, D. L. (2016). Ethical Issues in Forensic Psychology and Psychiatry. Ethics Med. Public Health 2 (1), 45-58. doi:10.1016/j.jemep.2016.01.015 
Smeijers, D., Bulten, E., Buitelaar, J., and Verkes, R.-J. (2018). Treatment Responsivity of Aggressive Forensic Psychiatric Outpatients. Int. J. Offender Ther. Comp. Criminol. 62 (12), 3834-3852. doi:10.1177/0306624x17747052

Smeijers, D., and Koole, S. L. (2019). Testing the Effects of a Virtual Reality Game for Aggressive Impulse Management (VR-GAIME): Study Protocol. Front. Psychiatry 10, 83. doi:10.3389/fpsyt.2019.00083

Sygel, K., and Wallinius, M. (2021). Immersive Virtual Reality Simulation in Forensic Psychiatry and Adjacent Clinical Fields: A Review of Current Assessment and Treatment Methods for Practitioners. Front. Psychiatry 12, 673089. doi:10.3389/fpsyt.2021.673089

Tapscott, J. L., Hancock, M., and Hoaken, P. N. S. (2012). Severity and Frequency of Reactive and Instrumental Violent Offending. Criminal Justice Behav. 39 (2), 202-219. doi:10.1177/0093854811429647

Tuente, S. K., Bogaerts, S., Van Ijzendoorn, S., and Veling, W. (2018). Effect of Virtual Reality Aggression Prevention Training for Forensic Psychiatric Patients (VRAPT): Study Protocol of a Multi-center RCT. BMC Psychiatry 18 (1), 251. doi:10.1186/s12888-018-1830-8

Völlm, B., Bartlett, P., and McDonald, R. (2016). Ethical Issues of Long-Term Forensic Psychiatric Care. Ethics Med. Public Health 2 (1), 36-44.

Warburton, W. A., and Anderson, C. A. (2015). Aggression, Social Psychology of. Int. Encyclopedia Soc. Behav. Sci. (Second Edi.) Vol. 1, 373-380. Elsevier. doi:10.1016/B978-0-08-097086-8.24002-6

Watt, B. D., and Howells, K. (1999). Skills Training for Aggression Control: Evaluation of an Anger Management Programme for Violent Offenders. Leg. Criminological Psychol. 4 (2), 285-300. doi:10.1348/135532599167914
Weech, S., Kenny, S., and Barnett-Cowan, M. (2019). Presence and Cybersickness in Virtual Reality Are Negatively Related: a Review. Front. Psychol. 10, 158. doi:10.3389/fpsyg.2019.00158

World Medical Association. (2013). WMA Declaration of Helsinki. Ethical Principles for Medical Research Involving Human Subjects. Available at: https://www.wma.net/policies-post/wma-declaration-of-helsinki-ethical-principlesfor-medical-research-involving-human-subjects.

Conflict of Interest: The authors declare that the research was conducted in the absence of any commercial or financial relationships that could be construed as a potential conflict of interest.

Publisher's Note: All claims expressed in this article are solely those of the authors and do not necessarily represent those of their affiliated organizations, or those of the publisher, the editors and the reviewers. Any product that may be evaluated in this article, or claim that may be made by its manufacturer, is not guaranteed or endorsed by the publisher.

Copyright (c) 2022 González Moraga, Klein Tuente, Perrin, Enebrink, Sygel, Veling and Wallinius. This is an open-access article distributed under the terms of the Creative Commons Attribution License (CC BY). The use, distribution or reproduction in other forums is permitted, provided the original author(s) and the copyright owner(s) are credited and that the original publication in this journal is cited, in accordance with accepted academic practice. No use, distribution or reproduction is permitted which does not comply with these terms. 